Frauen - Gesellschaft - Kritik

Band 41 


\section{Erfahrungen und \\ gesundheitliche Entwicklungen \\ lesbischer Frauen \\ im Coming-out-Prozess}

Gisela Wolf

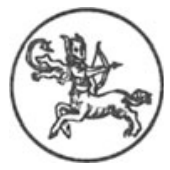

Centaurus Verlag \& Media UG 2004 
Der Druck der Dissertation wurde aus dem Dissertationenfonds der Universität Basel (Schweiz) gefördert.

Die Deutsche Bibliothek - CIP-Einheitsaufnahme

Wolf, Gisela:

Erfahrungen und gesundheitliche Entwicklungen lesbischer Frauen im Coming-out-Prozess / Gisela Wolf. Herbolzheim : Centaurus-Verl., 2004

(Frauen, Gesellschaft, Kritik; Bd. 41)

Zugl.: Basel, Univ., Diss., 2003

ISBN 978-3-8255-0427-4 ISBN 978-3-86226-392-9 (eBook)

DOI 10.1007/978-3-86226-392-9

\section{ISSN 0939-4540}

Alle Rechte, insbesondere das Recht der Vervielfältigung und Verbreitung sowie der Übersetzung, vorbehalten. Kein Teil des Werkes darf in irgendeiner Form (durch Fotokopie, Mikrofilm oder ein anderes Verfahren) ohne schriftliche Genehmigung des Verlages reproduziert oder unter Verwendung elektronischer Systeme verarbeitet, vervielfältigt oder verbreitet werden.

(c) CENTAURUS Verlags-GmbH \& Co. KG, Herbolzheim 2004

Satz: Vorlage der Autorin

Umschlaggestaltung: DTP-Studio, Antje Walter, Hinterzarten 


\section{Danksagung}

Meinen Dank möchte ich allen aussprechen, die diese Arbeit unterstützt haben. Zuerst möchte ich meinen Interviewpartnerinnen danken, die mir ihre Erfahrungen im Coming-out mitgeteilt und sich intensiv mit dieser Arbeit auseinander gesetzt haben. Sie hatten den Mut, einer Veröffentlichung auch von sehr tiefgehenden Erinnerungen zuzustimmen. Ich habe viel in unseren Gesprächen lernen können.

Mein Erstbetreuer, Herr Professor Dr. Udo Rauchfleisch, hat sich nach meiner langen Odyssee durch universitäre Systeme sofort zur Annahme des Themas bereit erklärt und den Abschlussprozess meiner Arbeit fachlich begleitet. Danken möchte ich hier auch meinem Zweitbetreuer, Herrn Dr. Gottfried Waser.

Während meiner Arbeit an der Dissertation habe ich fachlichen Rückhalt vor Ort in Freiburg durch Frau Professorin Dr. Nina Degele (Zentrum für Anthropologie und Gender Studies an der Universität Freiburg) erhalten. Ihr möchte ich besonders für ihre spontane Bereitschaft zur Diskussion und ihre vielfältige Unterstützung danken. Thre konstruktive Kritik hat noch viele der in dieser Arbeit dargestellten Inhalte geklärt, verschärft und verbessert.

Lesbische Frauen haben mir immer wieder Möglichkeiten geboten, Konzeption und Inhalte der Arbeit vorzustellen und zu diskutieren. Danken möchte ich auch besonders denjenigen Lesben, die für ihre Auseinandersetzungen mit VertreterInnen des Gesundheitssystems Ergebnisse meiner Arbeit bereits aufgegriffen haben. Ich bin gespannt, wie die Arbeit jetzt auch und gerade innerhalb von FrauenLesbenzusammenhängen aufgenommen wird.

Rückhalt für diese Arbeit habe ich in der Freiburger FrauenLesbenszene gefunden, im bestärkenden Zusammensein mit anderen frauenbezogenen Frauen und auch mit einigen schwulen Männern. In der politischen, beratenden und pädagogischen Projektearbeit kämpfen wir für ein Ziel, für das ich auch in dieser Arbeit eintreten möchte. Manchmal streiten wir, was uns und unsere Arbeit jedoch auch weiterbringen kann. Ich sehe diese Dissertation auch als eine Zurückgabe dessen, was ich innerhalb dieser Zusammenhänge mitbekommen habe.

Danken möchte ich auch meiner WG. Yann für den allmorgendlichen Wettkampf, wer von uns zuerst aufsteht, Birgit, Katharina, Valérie und Isa für die erbaulichen Gespräche am Küchentisch, bei denen es um alles andere jenseits dieser Arbeit ging und Birgit und Katharina auch besonders für das gemeinsame Aus-der-Puste-geraten beim reflexionsreichen gemeinsamen Joggen.

Meine Partnerin Cornelia hat mir immer wieder die Energie und auch die Leichtigkeit gegeben, im Blick zu behalten, was das Wichtigste im Leben ist. Schließlich ist sie wesentlich dafür verantwortlich, dass ich jeden Tag Lust aufs Leben und auf die gute, untrennbare Mischung aus Projektearbeit, Schreiben, Schaffen und freier Zeit habe. 
1 GRUNDLEGENDES ZUR VORLIEGENDEN UNTERSUCHUNG 15

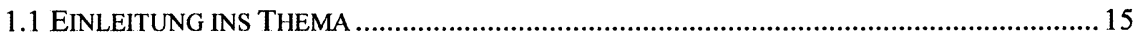

1.2 GRUNDSÄTZLICHES ZUR SPRACHWAHL UND BEGRIFFSERKLÄRUNGEN.......................16

1.3 FORSCHUNGSSTAND UND VERFÜGBARKEIT DER VORLIEGENDEN FORSCHUNG ...........25

1.4 DER FORSCHUNGSANSATZ DER VORLIEGENDEN UNTERSUCHUNG ...........................27

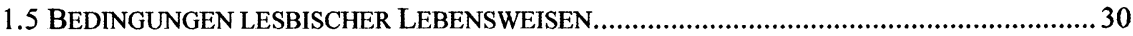

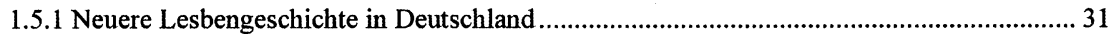

1.5.2 Sozialisationsbedingungen von Mädchen und Frauen ................................................... 48

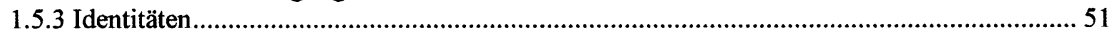

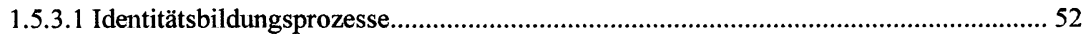

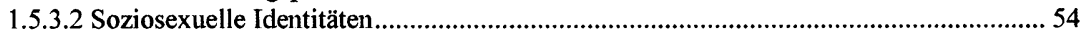

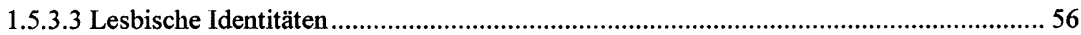

1.5.3.4 Lesbische Coming-out-Prozesse als Entwicklungsprozesse lesbischer Identitäten....... 59

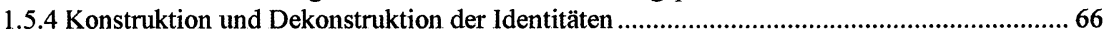

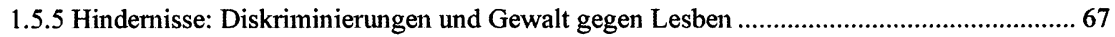

1.5.5.1 Die Grundlagen gesellschaftlicher und staatlicher Repressionen gegen Lesben ............68 68

1.5.5.2 Täter antilesbischer Diskriminierung und Gewalt ..................................................... 71

1.5.5.3 Erfahrungen lesbischer Frauen und Mädchen mit Diskriminierungen und Gewalt....... 73

1.5.5.4 Diskriminierungen und Gewalt gegen Lesben am Arbeitsplatz ................................... 78

1.5.5.5 Diskriminierungen und Gewalt gegen Lesben im medizinischen/psychologischen

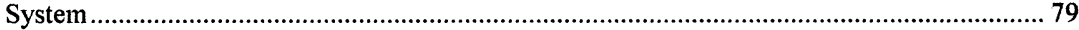

1.5.5.6 Diskriminierungserfahrungen von Lesben in christlich-religiös geprägten

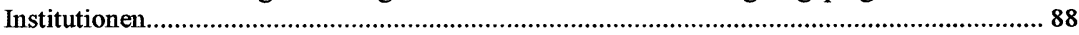

1.5.5.7 Folgen von Diskriminierungen und Gewalt gegen Lesben ....................................... 90

1.5.5.8 Gesundheitliche Beeinträchtigungen lesbischer Frauen als Folgen der Belastungen

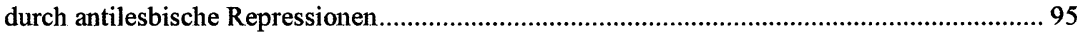

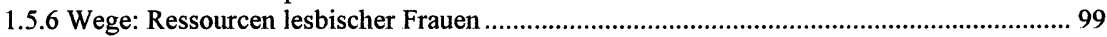

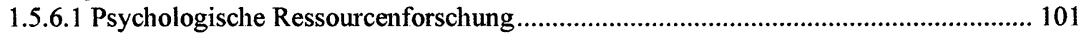

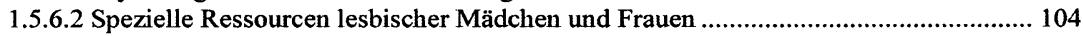

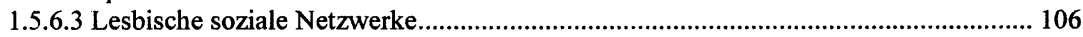

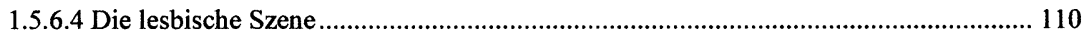

1.5.7 Lesbische Lebensweisen zwischen Verstecktleben und Offenleben................................ 113

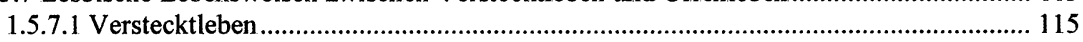

1.5.7.2 Zusammenhänge zwischen Verstecktleben und Gesundheit und Befinden................... 118

1.5.7.3 Selektives Verstecktleben und Offenleben.................................................................. 120

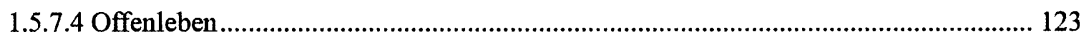

1.5.7.5 Zusammenhänge zwischen Offenleben und Gesundheit und Befinden ....................... 125

1.6 THEORETISCHE ANSÄTZE UND MODELLE ZU GESUNDHEITLICHEN

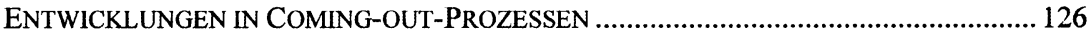

1.6.1 Das Konzept des Minoritäten-Stress von Virginia R. Brooks (1981) ............................... 127

1.6.2 Die „Minoritäts-Stress-Theorie“ nach Ilan Meyer (1995) und die Weiterentwicklung

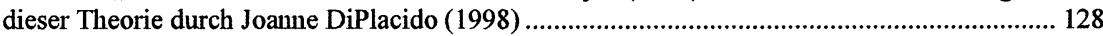

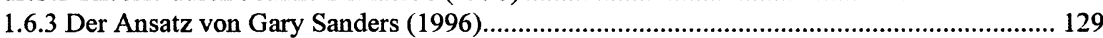

1.6.4 Modell zu gesundheitlichen Entwicklungen lesbischer Frauen im Coming-out-Prozess... 130 
2.1 DIE BEZIEHUNG ZWISCHEN INTERVIEWPARTNERINNEN UND FORSCHERIN.

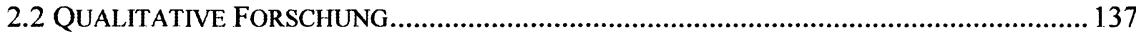

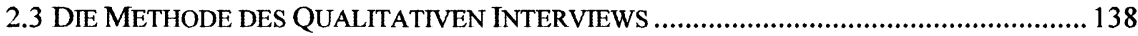

2.4 ALLGEMEINE DARSTELLUNG DER AUSWERTUNG DURCH QUALITATIVE

INHALTSANALYTISCHE VERFAHREN

2.5 DAS IN DER VORLIEGENDEN ARBEIT ENTWICKELTE INHALTSANALYTISCHE

VERFAHREN.

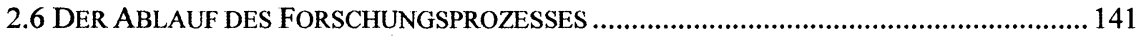

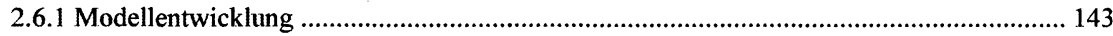

2.6.2 Fragestellungen und Thesen der vorliegenden Untersuchung ....................................... 143

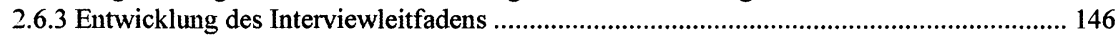

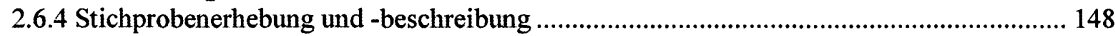

2.6.5 Beschreibung und sozioökonomische Daten der Interviewpartnerinnen ......................... 149

2.6.6 Kurzcharakterisierung der Interviewpartnerinnen ..................................................... 151

2.6.7 Durchführung der Interviews ................................................................................ 152

2.6.8 Protokollierung und Auswertung der Interviewatmosphäre und der Erfahrungen mit

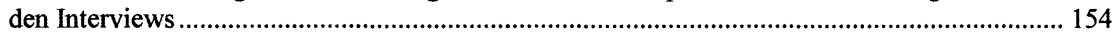

2.6.9 Transkription und Bearbeitung der Interviews............................................................... 157

2.6.10 Fundstellenkodierung und Herausarbeiten von zentralen Aussagen der

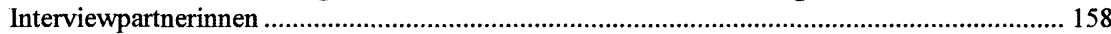

2.6.11 Kategorisierung und zeitliche Einordnung der Aussagen der Interviewpartnerinnen ...... 158 2.6.12 Analyse von thematisch zusammenhängenden Aussagen der Interviewpartnerinnen und Herausarbeiten von hypothetischen Zusammenhängen in Bezug auf gesundheitliche Entwicklungsprozesse.

2.6.13 Rückmeldung an die Interviewpartnerinnen und Überprüfung des Auswertungsprozedere

2.6.14 Zusammenstellung und inhaltliche Einordnung der ausgewerteten Aussagen der Interviewpartnerimnen in den Text der Arbeit

2.6.15 Analyse, Darstellung und Interpretation der Ergebnisse und Weiterentwicklung des Modells

2.6.16 Rückmeldung des gesamten Textes an die Interviewpartnerinnen und Auswertung

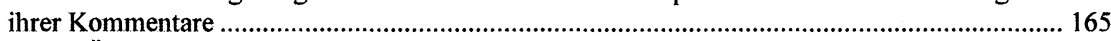

2.6.17 Überprüfung der Gütekriterien .................................................................................... 166

2.6.18 Bewertung des Untersuchungsverfahrens ........................................................................ 166

3 ERFAHRUNGEN DER INTERVIEWTEN LESBISCHEN FRAUEN ......................167

3.1 VERORTUNG DER ERFAHRUNGEN DER INTERVIEWPARTNERINNEN IN DER

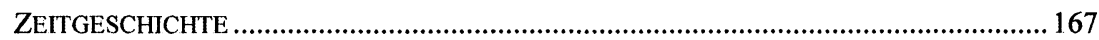

3.2 COMING-OUT-PROZESSE DER INTERVIEWTEN LESBISCHEN FRAUEN ........................ 168

3.2.1 Zur Selbstdefinition der Interviewpartnerinnen ........................................................... 169

3.2.2 Coming-out-Prozesse der interviewten lesbischen Frauen................................................ 170

3.3 KONSTRUKTION UND DEKONSTRUKTION DER IDENTITÄTEN AUS DER

PERSPEKTIVE DER INTERVIEWTEN LESBISCHEN FRAUEN......................................... 182

3.4 ERFAHRUNGEN DER INTERVIEWTEN LESBISCHEN FRAUEN MIT

DISKRIMINIERUNGEN UND GEWALT UND DIE FOLGEN ......................................... 186

3.4.1 Erinnerungen der Interviewpartnerinnen an Diskriminierungen und Gewalt vor dem Coming-out

3.4.2 Erinnerungen der Interviewpartnerinnen an Diskriminierungen und Gewalt während des inneren Coming-out und der inneren verstärkten Auseinandersetzung mit ihrer eigenen sozio-sexuellen Identität. 
3.4.3 Erinnerungen der Interviewpartnerinnen an Diskriminierungen und Gewalt während des äußeren Coming-out/Going-public

3.5 DIE RESSOURCEN DER BEFRAGTEN LESBISCHEN FRAUEN IM

COMING-OUT-PROZESS.

3.5.1 Ressourcen der befragten lesbischen Frauen vor dem inneren Coming-out....................... 209

3.5.2 Ressourcen der Interviewpartnerinnen im inneren Coming-out.

3.5.3 Ressourcen der Interviewpartnerimnen während der verstärkten inneren Auseinandersetzung mit ihrer lesbischen soziosexuellen Identität

3.5.4 Ressourcen der Interviewpartnerimnen während des äußeren Coming-out/Going-public .. 214

3.5.5 Perspektiven der Interviewpartnerinnen zum Interviewzeitpunkt ...................................... 219

3.5.6 Empfehlungen der Interviewpartnerinnen an Lesben im Coming-out-Prozess................... 223

3.5.7 Die lesbische Szene aus der Perspektive der interviewten lesbischen Frauen.................... 231

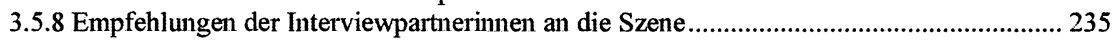

3.6 ERFAHRUNGEN UND LEBENSWEISEN DER INTERVIEWTEN LESBISCHEN FRAUEN ZWISCHEN DEN POLEN VERSTECKTLEBEN UND OFFENLEBEN ...............................236

3.7 GESUNDHEITLICHE ENTWICKLUNGEN DER INTERVIEWTEN LESBISCHEN FRAUEN IM COMING-OUT-PROZESS

3.7.1 Gesundheitliche Entwicklungen in der Kindheit der Interviewpartnerinnen ..... 243

3.7.2 Gesundheitliche Entwicklungen in der Pubertät der Interviewpartnerinnen und während ihres Übergangs ins Erwachsenenalter

3.7.3 Gesundheitliche Entwicklungen der Interviewpartnerinnen während der intensiven

Auseinandersetzungen mit ihrer soziosexuellen Identität im frühen Erwachsenenalter.....

3.7.4 Gesundheitliche Entwicklungen der Interviewpartnerinnen während ihres äußeren

Coming-out/Going-public im frühen Erwachsenenalter ........................................................ 260

3.7.5 Gesundheitliche Perspektiven der Interviewpartnerinnen zum Interviewzeitpunkt ........... 272

3.8 ZUSAMMENFASSUNG DER GESUNDHEITLICHEN ENTWICKLUNGEN DER BEFRAGTEN

LESBISCHEN FRAUEN IM COMUNG-OUT-PROZESS................................................... 279

4 DISKUSSION DER EMPIRISCHEN BEFUNDE

4.1 VERBINDUNGEN ZWISCHEN DEN VON DEN INTERVIEWPARTNERINNEN BERICHTETEN GESUNDHEITLICHEN ENTWICKLUNGEN UND BEFUNDEN AUS DER

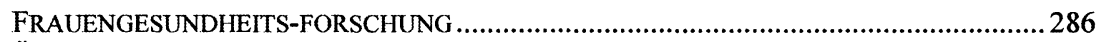

4.2 ÜBERPRÜFUNG DER FRAGESTELLUNGEN UND AUSGANGSTHESEN DER ARBEIT ........295

4.3 PRÜFUNG UND WEITERENTWICKLUNG DER THEORETISCHEN ANSÄTZE BEZÜGLICH GESUNDHEITLICHER ENTWICKLUNGEN BEI LESBISCHEN FRAUEN IM COMING-OUT-PROZESS..

4.3.1 Verstecken und Offenleben der lesbischen soziosexuellen Identität im Zusammenhang mit gesundheitlichen Entwicklungsprozessen

4.3.2 Geschlechtsspezifische Perspektiven

4.3.3 Der Stellenwert der Ressourcen im Coming-out-Prozess 301

4.3.4 Das weiterentwickelte Modell zu gesundheitlichen Entwicklungen lesbischer Frauen im Coming-out-Prozess.

4.4 GESELLSCHAFTSPOLITISCHE UND INSTITUTIONENBEZOGENE REFORM-

FORDERUNGEN.

4.4.1 Veränderungsbedarf innerhalb medizinischer und psychotherapeutischer Systeme ........... 309

4.4.1.1 Empfehlungen der Interviewpartnerinnen für Reformen innerhalb des

Gesundheitswesens

4.4.1.2 Handlungsmöglichkeiten lesbischer Frauen im Gesundheitssystem .......................... 310

4.4.1.3 Professionelle Leitlinien für Reformen innerhalb des Gesundheitssystems ................ 312

4.4.2 Lesbengerechte Beratungs- und Therapieangebote ............................................................. 316

4.4.2.1 Leitlinien und Aufgaben lesbengerechter Beratung und Therapie ............................. 317 


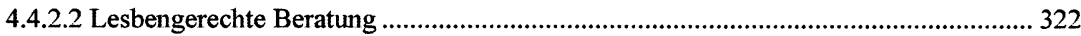

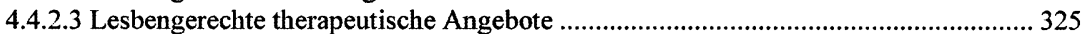

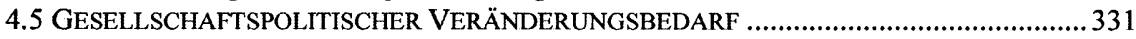

4.6 KONTEXTUALISIERUNG DER GESELLSCHAFTSPOLITISCHEN REFORM-

FORDERUNGEN DER INTERVIEWTEN LESBISCHEN FRAUEN ................................... 332

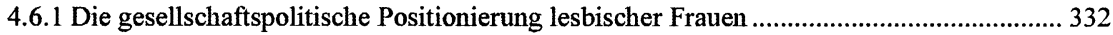

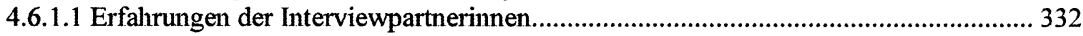

4.6.1.2 Kontextualisierung der Erfahrungen der Interviewpartnerinnen und Ableitung

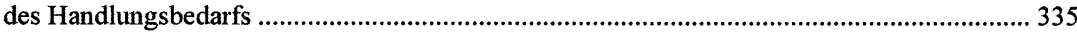

4.6.2 Die Arbeit in Schulen und anderen Einrichtungen für Kinder und Jugendliche ................ 337

4.6.2.1 Erfahrungen der Interviewpartnerinnen................................................................... 337

4.6.2.2 Kontextualisierung der Erfahrungen der Interviewpartnerinnen und Ableitung

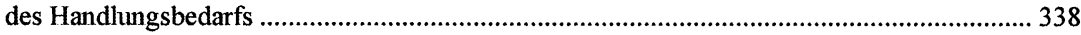

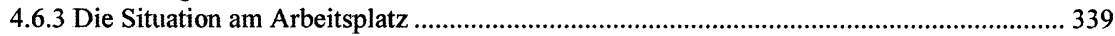

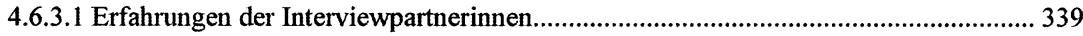

4.6.3.2 Kontextualisierung der Erfahrungen der Interviewpartnerinnen und Ableitung

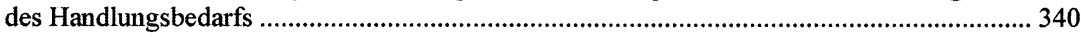

4.6.4 Forderungen nach rechtlicher Gleichstellung und nach einem Antidiskriminierungs-

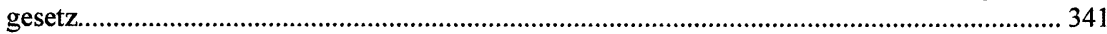

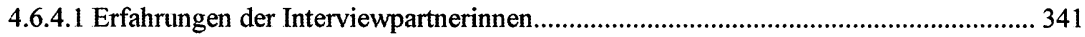

4.6.4.2 Kontextualisierung der Erfahrungen der Interviewpartnerinnen und Ableitung

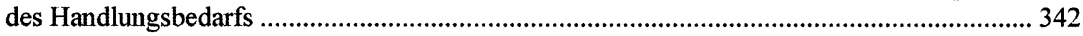

4.6.5 Grenzen bestehender rechtlicher Möglichkeiten zur Abwehr antilesbischer

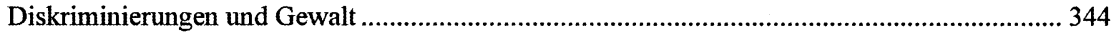

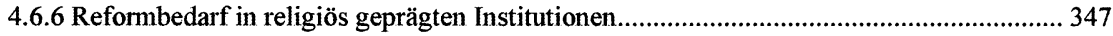

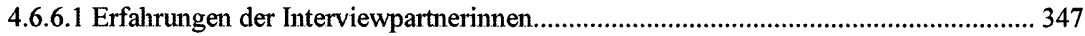

4.6.6.2 Kontextualisierung der Erfahrungen der Interviewpartnerinnen und Ableitung

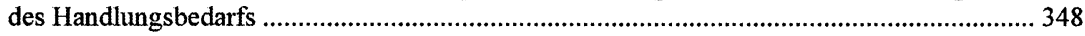

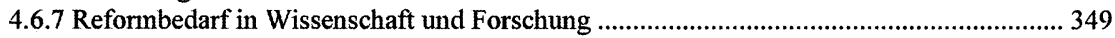

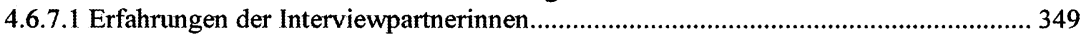

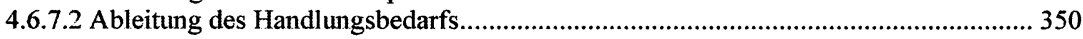

4.7 WECHSELWIRKUNGEN ZWISCHEN DEN REFORMFORDERUNGEN DER INTERVIEWPARTNERINNEN IN UNTERSCHIEDLICHEN GESELLSCHAFTSPOLITISCHEN

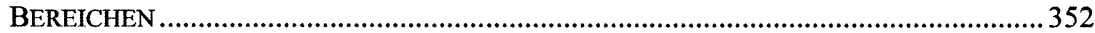

4.8 ABSCHLIEBENDE BEWERTUNGEN UND PERSPEKTIVEN DIESER ARBEIT ...................... 353

4.8.1 Bewertung und Perspektiven der Forschungsmethodik dieser Arbeit................................ 354

4.8.2 Bewertung und Perspektiven der empirischen Ergebnisse dieser Arbeit ........................... 361

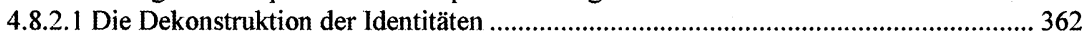

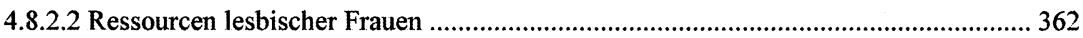

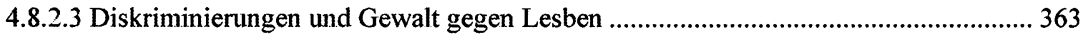

4.8.2.4 Gesundheitliche Entwicklungen im Coming-out-Prozess ......................................... 364

4.8.2.5 Gesellschaftspolitischer Veränderungsbedarf ......................................................... 365

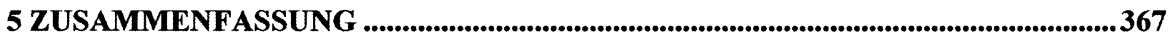

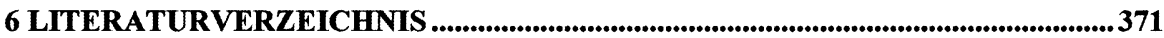

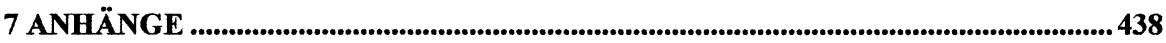

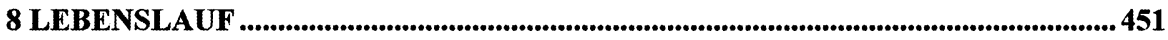




\section{Vorwort}

„Ich (...) glaube, dass es (...) zwar inzwischen viel Literatur der verschiedensten Art von Lesben für Lesben (gibt), ich (bin) aber nach wie vor der Auffassung (...) dass es noch zu wenig ist. Das ist vielleicht auch so 'n Punkt, was Frauen (...) auch machen können: Das Thema bearbeiten und irgendwie in die Welt setzen (...) weil ich glaube, so was ist unterstützend (...) für die, die nachkommen. "

(Interviewpartnerin Sophia, 28. 12. 1999)

Ich war einigermaßen erstaunt, als ich vor zwei Jahren bei meiner Suche nach Forschung zu lesbischen und schwulen Lebensweisen auf die Untersuchung von Gary Sanders (1996) stieß und dort las, wie ein schwuler Mann im Verlauf seines Comingout entscheidende Verbesserungen seiner Grand-mal-Epilepsie erleben konnte. Zwar hatte ich immer wieder Berichte von Lesben und Schwulen gehört, die nach ihrem Coming-out neben erheblichen Veränderungen in zentralen Lebensbereichen auch gesundheitsbezogene Entwicklungen erlebt hatten. Allerdings wunderte ich mich darüber, dass sich jemand dieses Themas als Forschungsgegenstand angenommen hatte und dies in einer renommierten Fachzeitschrift (System Familie) publizierte. War ich doch selbst während meines Studiums, das mich zur Psychologin qualifizierte, kaum auf Wissen über die Existenz lesbischer Mädchen und Frauen (oder schwuler Jungen und Männer) gestoßen, geschweige denn, dass ich mit den fachlichen Bedingungen von Lesbenforschung vertraut gemacht worden wäre. Die einzigen allen Studierenden meines Fachbereichs zum Thema vermittelten Bildungsinhalte waren diverse homophobe Bemerkungen in Lehrbüchern, welche in Freiburg bereits seit Jahren als Prüfungsliteratur dienten (z.B. Hoffmann \& Hochapfel 1991, Kutter 1989).

$\mathrm{Da}$ ich die gesellschaftliche Marginalisierung und das Verschweigen meiner eigenen soziosexuellen Identität selbst aufgenommen hatte, war mir zunächst das inneruniversitäre Verschweigen und Stigmatisieren lesbischer Lebensweisen kaum aufgefallen. Erst mehrere Jahre nach Abschluss meines Studiums, nach meinem eigenen Goingpublic und politischen Lernerfahrungen in lesbischen Zusammenhängen entwickelte sich meine Wahrnehmung dafür, was fehlte und was verschwiegen wurde und immer noch wird. So gehe ich heute auch davon aus, dass das Vertuschen und in die Unseriosität Abdrängen aller alternativ zur dominierenden Heterosexualität gelebter Lebensentwürfe gesellschaftliche Funktionen im Sinne einer Stabilisierung von bestehenden Privilegierungsstrukturen hat. Lesbengerechte Forschung betrachte ich als eine Möglichkeit der Dokumentation der Erfahrungen lesbischer Frauen. Zur Verbesserung des gesamtgesellschaftlichen Ist-Zustands müssen weitere Schritte folgen. 
Zum Entstehen der vorliegenden Arbeit haben dann noch mehrere weitere Faktoren beigetragen:

- die Unterstützung aus der Community und der Erfahrungsaustausch mit vielen anderen Lesben und bisexuellen Frauen sowie auch einigen schwulen Männern,

- meine im Austausch mit KollegInnen (insbesondere innerhalb des Berufsverbandes lesbischer Psychologinnen und schwuler Psychologen e.V.-VLSP-) und durch eigene Literaturrecherche vorangetriebene Weiterbildung in Bezug auf Inhalte und Methodiken der Lesbenforschung,

- meine Erfahrungen in der (unbezahlten) Beratungsarbeit mit Lesben und der antidiskriminatorischen Aufklärungsarbeit im Team mit anderen engagierten Lesben und Schwulen während der letzten acht Jahre,

- und die Freiheiten, die mir meine finanzielle Absicherung durch eine Vielfalt von Nebenjobs und durch Versicherungsgelder nach einem Unfall eröffnete.

Ich freue mich, wenn diese Arbeit Auseinandersetzungen und Weiterentwicklungen auslösen kann. Für Rückmeldungen, Kritik und Anmerkungen bin ich offen. 